BMJ Open Sport \& Exercise Medicine

\section{Effect of short and long moderate- intensity exercises in modifying cardiometabolic markers in sedentary Kenyans aged 50 years and above}

To cite: Magutah K, Meiring R, Patel NB, et al. Effect of short and long moderate-intensity exercises in modifying cardiometabolic markers in sedentary Kenyans aged 50 years and above. BMJ Open Sport \& Exercise Medicine 2018:4:e000316. doi:10.1136/ bmjsem-2017-000316

Accepted 12 March 2018
Check for updates

${ }^{1}$ Department of Medical Physiology, University of Nairobi, Nairobi, Kenya

${ }^{2}$ Department of Medical Physiology, Moi University, Eldoret, Kenya

${ }^{3}$ Exercise Physiology Laboratory, Faculty of Health Sciences, School of Physiology, University of the Witwatersrand, Johannesburg, South Africa

Correspondence to Dr Karani Magutah; kmagutah@ yahoo.com

\section{ABSTRACT}

Objectives We compared effects of shorter moderateintensity exercise time ( $<10$ min bouts) on cardiometabolic parameters with the current recommendations among elderly adults.

Methods Fifty-three sedentary individuals aged $\geq 50$ years were divided into exercise groups ${ }^{1}$ : male and ${ }^{2}$ female short-duration bouts ( $\mathrm{M}_{\mathrm{S}}$ and $\mathrm{F}_{\mathrm{S}}$, respectively), and $^{3}$ male and ${ }^{4}$ female long-duration bouts ( $M_{L}$ and $F_{L}$, respectively). Short-duration bouts consisted three 5-10 min moderate-intensity jogging sessions daily, and long-duration bouts consisted 30-60 min sessions 3-5 days weekly. Cumulative exercise times were equivalent. Physical activity (PA) was measured by log and activity monitors. Fasting venous blood at baseline and 8 weekly intervals was used for blood chemistry.

Results After 24 weeks, $M_{\mathrm{S}}$ and $\mathrm{F}_{\mathrm{S}}$ with total cholesterol (TC) of $>5.2 \mathrm{mmol} / \mathrm{L}$ and $>5.3 \mathrm{mmol} / \mathrm{L}$ decreased from $22.2 \%$ to $14.8 \%$ and from $30.9 \%$ to $11.5 \%$, respectively. For $M_{L}$, this decreased from $25.9 \%$ to $3.7 \%$, while $F_{L}$ had $0 \%$ change. In $M_{S}$ and $M_{1}, T C /$ high-density lipoproteins (HDLs) of $>5.0 \mathrm{mmol} / \mathrm{L}$ dropped from $22.2 \%$ to $7.4 \%$ and from $22.2 \%$ to $15.4 \%$, respectively. In $F_{S}$ and $F_{L}, T C /$ $\mathrm{HDL}$ of $>4.5 \mathrm{mmol} / \mathrm{L}$ declined from $19.2 \%$ to $7.7 \%$ and from $19.2 \%$ to $3.8 \%$, respectively. $M_{S}$ and $M_{L}$ with fasting blood glucose of $\geq 5.5 \mathrm{mmol} / \mathrm{L}$ declined from $40.7 \%$ to $11.1 \%$ and from $33.3 \%$ to $3.7 \%$, respectively. Similarly, it declined from $46.2 \%$ to $0 \%$ and $42.3 \%$ to $11.5 \%$ for $F_{S}$ and $F_{L}$, respectively. There were no differences in the changes between regimes throughout the study. Conclusion Bouts lasting $<10$ min per session are as good as those lasting $; \geq 30$ min in improving cardiometabolic profiles of sedentary adults aged $\geq 50$ years.

\section{INTRODUCTION}

The current recommendation for weekly duration of moderate-intensity PA for health benefit in adults is a minimum of $150 \mathrm{~min}$, traditionally achieved in 3-5 sessions of 30-60 min per week, or accumulated through bouts that last more than $10 \mathrm{~min}^{1}$ In some developed economies, $51 \%-79 \%$ of adults do not meet this weekly PA recommendation. ${ }^{2}$ In Eldoret, Kenya, where the current study
What are the new findings?

Majority of sedentary Kenyans aged above 50 years have metabolic health risks.

- Moderate-intensity exercise offered in $<10 \mathrm{~min}$ bouts has similar effects to longer bouts in modifying cardio-metabolic markers in sedentary adults.

- Regardless of the sex, accumulated short bouts of moderate-intensity exercise that last less than 10 min have beneficial health effects.

was performed, more than $82 \%$ of elderly adults do not achieve recommended levels of exercise. ${ }^{3}$ A lack of time due to other responsibilities partly contributes to lack of adherence to this PA recommendation. ${ }^{1-6}$ To improve adherence, it has been recommended that more experimental studies on the intensity, frequency and duration of exercise be undertaken and that would have a more global appeal on the benefits of exercise ${ }^{1-11}$ and, in particular, studies that lead to more appealing exercise regimes for older adults.

In the 1990s, suggestions were made that short exercise bouts were as effective as single continuous sessions of similar intensities provided that the cumulative weekly exercise time is equal. ${ }^{12}$ However, evidence for this suggestion remains inconclusive as existing guidelines still focus on either single continuous sessions or bouts that last at the very least $10 \mathrm{~min}$. This has thus failed to mitigate the poor exercise adherence observed among the older populace. ${ }^{13}$ Studies on the efficacy of even shorter exercise regimes that last less than 10 min remain scarce, leading the WHO to highlight the need for further studies on effectiveness of short-duration exercise. ${ }^{1}$

The cardiometabolic health benefits received from exercise for the elderly is clear, with a strong linkage to improved cardiorespiratory fitness (CRF) ${ }^{14-18}$ but the specific type of exercise regime that shows higher adherence yet yields similar cardiometabolic 
benefits needs to be determined. Several studies ${ }^{12} 1920$ have reported benefits of accumulated shorter exercise bouts. The lack of longer term follow-up in these studies has raised the question of the long-term benefits ${ }^{21-23}$ the short bouts may accrue. Therefore, in this study, cardiometabolic markers were compared for 24 weeks between sedentary elderly adults undertaking long-duration exercise bouts lasting $\geq 30 \mathrm{~min}$ and those performing short-duration exercise bouts that lasted $<10 \mathrm{~min}$.

\section{MATERIALS AND METHODS Participants}

Healthy sedentary males $(n=27)$ and females $(n=26)$ aged $\geq 50$ years were recruited from Eldoret, Kenya, following a local print advertisement. Sedentariness was defined as exercise/activity amounting to $<600$ metabolic equivalent minutes, using the WHO Global Physical Activity Questionnaire (GPAQ). A physical examination to rule out existing health problems was performed on all volunteers on their verbal declaration they were in good health. Individuals with cardiorespiratory disease or other physical ailments/injuries were excluded. Also excluded were participants on $\beta$-blockers and participants with diabetes on therapy. Signed informed consent was obtained from all participants.

\section{Protocol}

A biodemographics questionnaire on exercise history was completed for each participant following which they were randomly allocated into four groups ${ }^{1}$ : male short-duration bouts of exercise $\left(\mathrm{M}_{\mathrm{S}}\right),{ }^{2}$ female short-duration bouts of exercise $\left(\mathrm{F}_{\mathrm{S}}\right),{ }^{3}$ male long-duration bouts of exercise $\left(M_{L}\right)$ and female long-duration bouts of exercise $\left(F_{L}\right)$. In the short-duration bouts of exercise groups, the participants engaged in three sessions of 5-10 min of moderate-intensity jogging daily. In the long-duration bouts of exercise groups, participants engaged in 30-60 min jogging sessions for 3-5 days each week. Data from the participants were collected and analysed at the recruitment stage (September 2016) and then at 8-week intervals for a period of 24 weeks after the start of the exercise intervention to culminate in May 2017. Participants kept an exercise log, and objective verification of adherence to the exercise regime was done weekly using Polar Wearlink ActITrainer Accelerometers (Actigraph, Pensacola, Florida, USA), and activity monitors were fastened onto participants on select days. The data, metabolic equivalent minutes achieved from the activity monitors, were analysed weekly to ascertain that participants in the different exercise regimes had comparable cumulative exercise time. Since this was only available for select days and used to primarily verify adherence, exercise intensity was monitored using WHO GPAQ. For clinical analysis, $4 \mathrm{~mL}$ of venous blood was collected from an average of two participants daily following an overnight 12 hours' fast, usually between 06:00 and 08:00. Two drops of fleshly drawn blood were immediately used to measure blood glucose using Freestyle
Optium Glucometer (Abbott, Oxfordshire, UK). For lipid profiles, centrifugation of blood was done within an hour of collection following which the serum was frozen and kept at $-12{ }^{\circ} \mathrm{C}$, awaiting to run accumulated weekly samples together. This analysis into total cholesterols (TCs), high-density lipoproteins (HDLs), low-density lipoproteins (LDLs) and triglycerides (TGs) was done using Cobas Integra 400 Plus (Roche, Germany) at the Academic Model Providing Access to Healthcare Reference Laboratories, a certified clinical laboratory run by Indiana University/Moi University partnership.

\section{Analysis}

Data analysis was done at univariate, bivariate and multivariate levels based on sex of participant and their exercise regime. Paired t-tests were used for intragender differences of metabolic markers following the different exercise regimes. The outcome variables were further analysed at the multivariate level by performing multiple analysis of variance for the dependent variables of lipid profiles and blood glucose and, further, linear regressions controlling for sexgroup performed. For comparison across the four phases of the experiment, repeated measures analysis of variance was conducted for equality of means of these blood chemistry results. Analysis was done using STATA V.13; p value was set at $\leq 0.05$.

\section{RESULTS}

The mean age of the males $(n=27)$ was $55.5 \pm 3.0$ years and $53.9 \pm 3.0$ years for the females $(n=26)$. The proportion of participants who had completed tertiary education was $88.4 \%$ among the male and $70.4 \%$ among the female. Ninety-one per cent of participants were in white-collar jobs. All $(100 \%)$ male and female participants in the $M_{S}$ $(n=14)$ and $F_{S}(n=13)$ group adhered to their 24-week exercise regime, compared with $61.5 \%$ of the $M_{L}(n=14)$ and $76.9 \%$ of $\mathrm{F}_{\mathrm{L}}(\mathrm{n}=13)$. The weekly cumulative exercise minutes was similar for $\mathrm{M}_{\mathrm{S}}$ and $\mathrm{M}_{\mathrm{L}}$ (161.8 \pm 7.2 vs $162.56 \pm 6.1$, respectively) and also for $\mathrm{F}_{\mathrm{S}}$ and $\mathrm{F}_{\mathrm{L}}(158.3 \pm 3.6$ vs $156.05 \pm 2.7$, respectively). Specific baseline characteristics for participants from the four groups are presented in table 1.

Mean fasting blood glucose (FBG) of all participants at the start of the study was $5.92 \pm 1.4 \mathrm{mmol} / \mathrm{L}$ (males) and $6.23 \pm 0.74 \mathrm{mmol} / \mathrm{L}$ (females). The percentage of males and females with FBG $>5.5 \mathrm{mmol} / \mathrm{L}$ was $40.7 \%\left(\mathrm{M}_{\mathrm{s}}\right.$ group), 33.3\% ( $\mathrm{M}_{\mathrm{L}}$ group), $46.2 \%$ ( $\mathrm{F}_{\mathrm{S}}$ group) and $42.3 \%$ $\left(\mathrm{F}_{\mathrm{L}}\right.$ group $)$. In $22.2 \%\left(\mathrm{M}_{\mathrm{S}}\right), 25.9 \%\left(\mathrm{M}_{\mathrm{L}}\right), 30.8 \%\left(\mathrm{~F}_{\mathrm{S}}\right)$ and $11.5 \%\left(\mathrm{~F}_{\mathrm{L}}\right)$, baseline $\mathrm{TC}$ was above the cut-off of $>5.2$ $\mathrm{mmol} / \mathrm{L}$ for males and $>5.3 \mathrm{mmol} / \mathrm{L}$ for females. HDLs below $0.9 \mathrm{mmol} / \mathrm{L}$ was found in $18.5 \%\left(\mathrm{M}_{\mathrm{S}}\right), 11.1 \%\left(\mathrm{M}_{\mathrm{L}}\right)$, $7.7 \%\left(\mathrm{~F}_{\mathrm{S}}\right)$ and $15.4 \%\left(\mathrm{~F}_{\mathrm{L}}\right)$. TC/ HDL ratio of $>5.0 \mathrm{mmol} / \mathrm{L}$ and $>4.5 \mathrm{mmol} / \mathrm{L}$, the cut-offs for males and females, respectively, was found in $44.4 \%$ of the males $(22.2 \%$ each in $\mathrm{M}_{\mathrm{S}}($ mean=6.58 \pm 1.21$)$ and $\mathrm{M}_{\mathrm{L}}($ mean=6.63 \pm 1.29$\left.)\right)$ and $38.5 \%$ of the females $\left(19.2 \%\right.$ each in $\mathrm{F}_{\mathrm{S}}($ mean $=5.38 \pm 0.92)$ and $\mathrm{F}_{\mathrm{L}}($ mean $\left.=5.42 \pm 0.59)\right)$. LDL/HDL ratio $>3.5$ was found in $14.8 \% \mathrm{M}_{\mathrm{S}}$ group (mean=4.07 \pm 0.53 ) and in 
Table 1 Baseline characteristics of participants in the four groups

\begin{tabular}{lllll}
\hline & MS & ML & FS & FL \\
\hline Age (years) & $55.0 \pm 5.6$ & $55.2 \pm 3.0$ & $53.9 \pm 2.6$ & $53.9 \pm 3.5$ \\
BMI $\left(\mathrm{kg} / \mathrm{m}^{2}\right)$ & $25.8 \pm 4.0$ & $28.6 \pm 4.8$ & $33.3 \pm 4.8$ & $32.0 \pm 5.4$ \\
Waist/height ratio & $0.52 \pm 0.07$ & $0.56 \pm 0.08$ & $0.61 \pm 0.05$ & $0.57 \pm 0.08$ \\
Waist/hip ratio & $0.93 \pm 0.06$ & $0.96 \pm 0.07$ & $0.82 \pm 0.10$ & $0.84 \pm 0.09$ \\
Fat \% & $20.7 \pm 7.3$ & $24.9 \pm 7.9$ & $39.8 \pm 3.6$ & $37.4 \pm 5.1$ \\
Resting systolic pressure $(\mathrm{mm} \mathrm{Hg})$ & $138.9 \pm 17.4$ & $140.8 \pm 22.1$ & $133.7 \pm 13.2$ & $137.6 \pm 23.8$ \\
Resting diastolic pressure $(\mathrm{mm} \mathrm{Hg})$ & $82.1 \pm 11.3$ & $83.7 \pm 8.7$ & $83.7 \pm 10.6$ & $84.2 \pm 8.1$ \\
Resting heart rate $(\mathrm{b} / \mathrm{m})$ & $73.9 \pm 9.5$ & $76.8 \pm 7.7$ & $79.8 \pm 12.0$ & $71.8 \pm 8.4$ \\
\hline
\end{tabular}

Data presented as mean \pm SD.

$\operatorname{BMI}\left(\mathrm{kg} / \mathrm{m}^{2}\right)$, basal metabolic index in kilograms per meter squared; $b / \mathrm{m}$, beats per minute; $F_{L}$, long bouts female; $F_{S}$, short bouts female; $M_{L}$, long bouts male; $\mathrm{M}_{\mathrm{S}}$, short bouts male.

$18.5 \% \mathrm{M}_{\mathrm{L}}$ group $($ mean $=4.79 \pm 1)$ LDL/HDL ratio $>3.0$ was found in $23.1 \% \mathrm{~F}_{\mathrm{S}}$ group (mean=3.53 \pm 0.27 ) and $11.1 \% \mathrm{~F}_{\mathrm{L}}$ group $($ mean $=3.32 \pm 0.43)$.

At the end of the 24-week period, male participants with $\mathrm{FBG}$ of $\geq 5.5 \mathrm{mmol} / \mathrm{L}$ in both the $\mathrm{M}_{\mathrm{S}}$ and $\mathrm{M}_{\mathrm{L}}$ groups decreased by $29.6 \%$. The percentage of female participants in $\mathrm{F}_{\mathrm{S}}$ group with $\mathrm{FBG}$ of $\geq 5.5 \mathrm{mmol} / \mathrm{L}$ dropped from $46.2 \%$ to 0 , while in $\mathrm{F}_{\mathrm{L}}$ group, it decreased by $30.8 \%$. Mean glucose values for all the groups also dropped, but there was no significant gender difference between different exercise regimes (figure 1). All $M_{S}$ and $F_{S}$ who had baseline $\mathrm{HDL}$ of $<0.9 \mathrm{mmol} / \mathrm{L}$ reached values of $\geq 0.9$ $\mathrm{mmol} / \mathrm{L}$, the recommended lower cut-off. There was no percentage change in the subjects in $\mathrm{M}_{\mathrm{L}}$ group with HDL $<0.9 \mathrm{mmol} / \mathrm{L}$. Seventy-five per cent of $\mathrm{F}_{\mathrm{L}}$ group reached $\mathrm{HDL} \geq 0.9 \mathrm{mmol} / \mathrm{L}$. For TC in the $\mathrm{M}_{\mathrm{S}}$ group, participants with $>5.2 \mathrm{mmol} / \mathrm{L}$ fell from $22.2 \%$ to $14.8 \%$, with mean value decreasing from $6.4 \pm 1.38 \mathrm{mmol} / \mathrm{L}$ to $5.48 \pm 0.41 \mathrm{mmol} / \mathrm{L}$. In the $\mathrm{M}_{\mathrm{L}}$ group, the reduction was from $25.9 \%$ to $3.7 \%$, with mean TC decreasing from
$6.09 \pm 0.58 \mathrm{mmol} / \mathrm{L}$ to $5.76 \mathrm{mmol} / \mathrm{L}$. Similarly, in $\mathrm{F}_{\mathrm{S}}$ group, participants with TC $>5.3 \mathrm{mmol} / \mathrm{L}$ decreased from $30.8 \%$ to $11.5 \%$ with the mean TC decreasing from $6.75 \pm 1.46$ to $5.72 \pm 0.15 \mathrm{mmol} / \mathrm{L}$. In $\mathrm{F}_{\mathrm{L}}$ group, there was no change in number of participants with TC $>5.3 \mathrm{mmol} / \mathrm{L}$, although their mean values decreased from $7.78 \pm 1.79 \mathrm{mmol} / \mathrm{L}$ to $5.76 \pm 0.44 \mathrm{mmol} / \mathrm{L}$. For the $\mathrm{TC} / \mathrm{HDL}$ ratio, in the $\mathrm{M}_{\mathrm{S}}$ group participants with ratios $>5.0$ decreased from $22.2 \%$ to $7.4 \%$ (mean decreased from $6.58 \pm 1.21$ to $5.59 \pm 0.36$ ), and in $\mathrm{F}_{\mathrm{S}}$ group, those with the ratio $>4.5$ decreased from $19.2 \%$ to $7.7 \%$ (mean decreased from $5.38 \pm 0.92$ to $5.14 \pm 0.03)$. In the $\mathrm{M}_{\mathrm{L}}$ group, the decrease was from $22.2 \%$ to $15.4 \%$ (mean change from $6.63 \pm 1.29$ to $5.66 \pm 0.5$ ), and in $\mathrm{F}_{\mathrm{L}}$ group, the decrease was from $19.2 \%$ to $3.8 \%$ (mean change from $5.42 \pm 0.59$ to $5.18 \pm 0$ ). Percentage of $\mathrm{M}_{\mathrm{S}}$ group with $\mathrm{LDL} / \mathrm{HDL}>3.5 \mathrm{mmol} / \mathrm{L}$ was halved to $7.4 \%$ with mean $\mathrm{LDL} / \mathrm{HDL}$ decreasing to $3.61 \pm 0.14$. In the $\mathrm{M}_{\mathrm{L}}$ group, the decrease was from $18.5 \%$ to $11.1 \%$ with mean LDL/HDL decreasing to $4.65 \pm 0.67 \mathrm{mmol} / \mathrm{L}$. In $\mathrm{F}_{\mathrm{S}}$ group, participants with LDL/HDL ratio $>3.0$ decreased

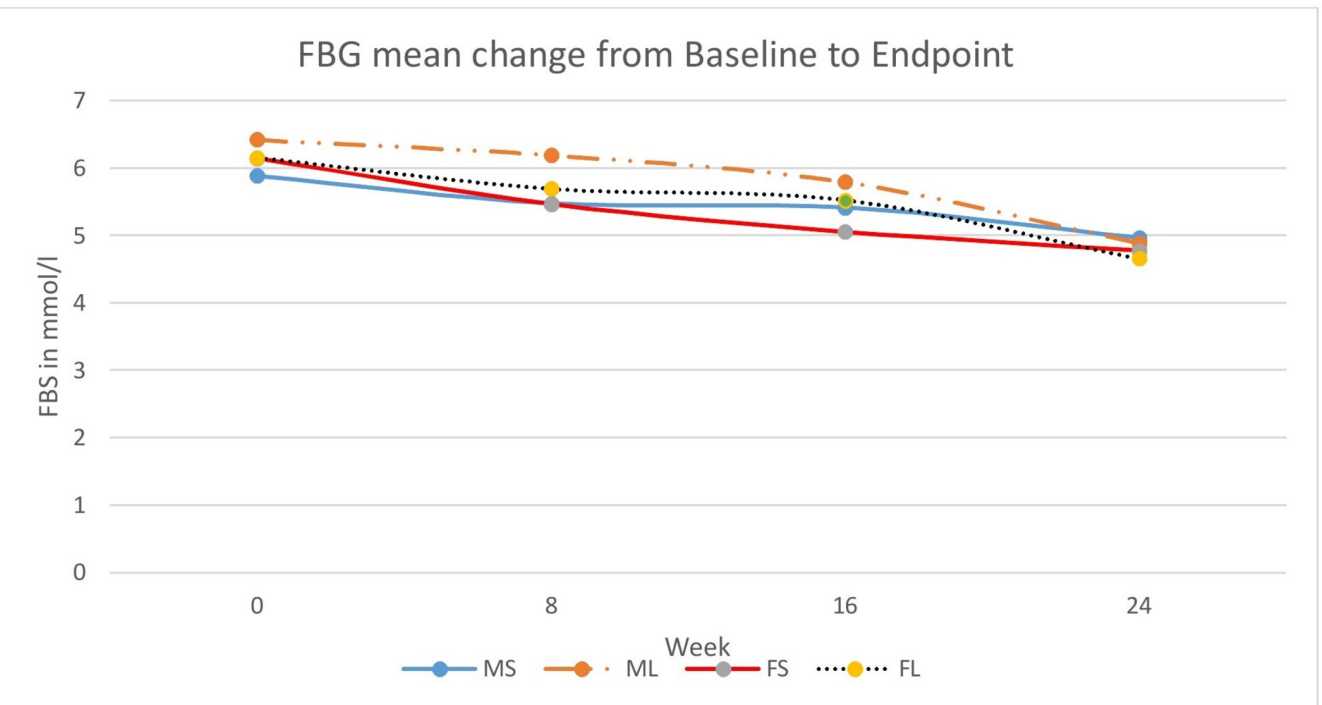

Figure 1 Mean change in fasting blood glucose (FBG) between week 0 and week 24. For each exercise regime, FB dropped over the 24-week period in both sexes. FBG, fasting blood glucose; FL, long bouts female; FS, short bouts female; ML, long bouts male; MS, short bouts male. 


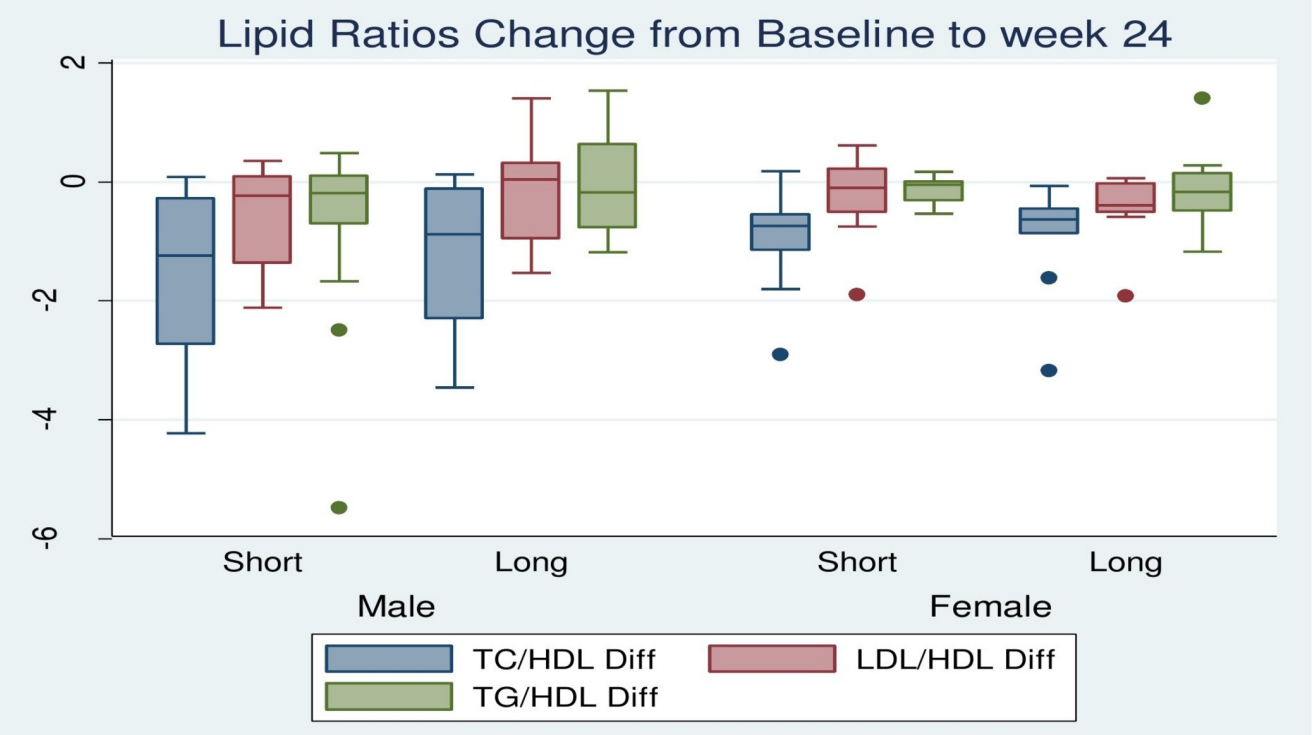

Figure 2 Mean change in lipid ratios between week 0 and week 24. No differences were observed in the change in lipid ratios in 24 weeks for the different exercise regimes in each either sex. HDL, high-density lipoprotein; LDL, low-density lipoprotein; TC, total cholesterol; TG, triglycerides.

from $23.1 \%$ to $15.4 \%$ (mean $3.49 \pm 0.55$ ) and to $3.8 \%$ from $11.1 \%$ (mean $3.49 \pm 0$ ) in the $\mathrm{F}_{\mathrm{L}}$ group. Mean changes for the lipid ratios are provided in figure 2.

In summary, except for TC in females where the short bouts appeared superior to the long bouts, there was no difference in actual value change between the two bouts for the various cardiometabolic parameters over the 24-week period as shown in table 2. Table 3 is linear regression outcomes controlling for gender in the difference of the means above.

\section{DISCUSSION}

\section{Baseline outcomes}

Lipid profiling is crucial in CRF assessment among healthy individuals and those with metabolic syndrome. At the start of the study, nearly half of the males and females had TC above their reference cut-offs $(>5.2$ and $>5.3 \mathrm{mmol} / \mathrm{L}$, respectively). A third of the males and females had unfavourable levels of both LDL and HDL. Additionally, one-fifth and one-tenth of males and females had high TG levels. For TC/HDL associated with poor CRF and cardiovascular disease, ${ }^{24-26}$ about half from both sexes had values higher than cut-off value. Similarly, a third of males and females had unfavourably high LDL/HDL, and about one-tenth had TG/HDL consistent with cardiometabolic risks. Current cut-offs used for TG/HDL ${ }^{25}$ are from non-black populations, and these may be different in other populations. Combination of various lipids and their ratios showed in the study participants a large proportion being at substantial metabolic risk at the start of the study. For FBG, participants had prediabetic to diabetic mean similar to those found in studies on other African populations of comparable ages, ${ }^{27} 28$ and mean values for females were higher than their age-matched males. Results from urban Nigerians and rural Kenyans ${ }^{29}{ }^{30}$ differ from the baseline measurement found in this study, probably because we studied a relatively older population. Thus, a majority of this urban population had metabolic health risks at recruitment, a finding similar to that recently found among rural Kenyans. ${ }^{30}$

\section{Absolute lipid profile changes}

After 24 weeks of prescribed moderate-intensity exercise, both males and females showed improved metabolic profiles. Nearly half of the $\mathrm{M}_{\mathrm{s}}$ group with unfavourable $\mathrm{TC}$ at the start reached values associated with better CRF. Furthermore, mean TC in these males decreased as did the mean for those who still did not reach the recommended ranges. The finding was similar among $M_{L}$ group, suggesting the two exercise regimes produced comparable affect. This $\mathrm{M}_{\mathrm{L}}$ group also had a reduced percentage that remained with values above recommended cut-off and a decline in mean values for the whole group and, importantly, those who did not attain TC values $<5.2 \mathrm{mmol} / \mathrm{L}$ as well. The two exercise regimes produced similar changes in the mean $\mathrm{TC}$ at the end of the study period. In females, except for the decrease in mean TC being higher in $\mathrm{F}_{\mathrm{S}}$ group, which suggests shortbout exercise could have better outcome compared with the currently recommended exercise regime, no other differences were observed in TC. Also, two-thirds of females with high baseline $\mathrm{TC}$ in $\mathrm{F}_{\mathrm{S}}$ group reached the recommended range at the end of the study; none from the $F_{L}$ group did. The two exercise regimes, however, had comparable decrease in the overall TC means, even among participants who were unable to achieve the reference cut-off. When baseline and end-point values were compared, mean TC change was similar in the two regimes. Thus, based on TC alone, no demonstrable 
Table 2 Mean cardiometabolic values over 24 weeks

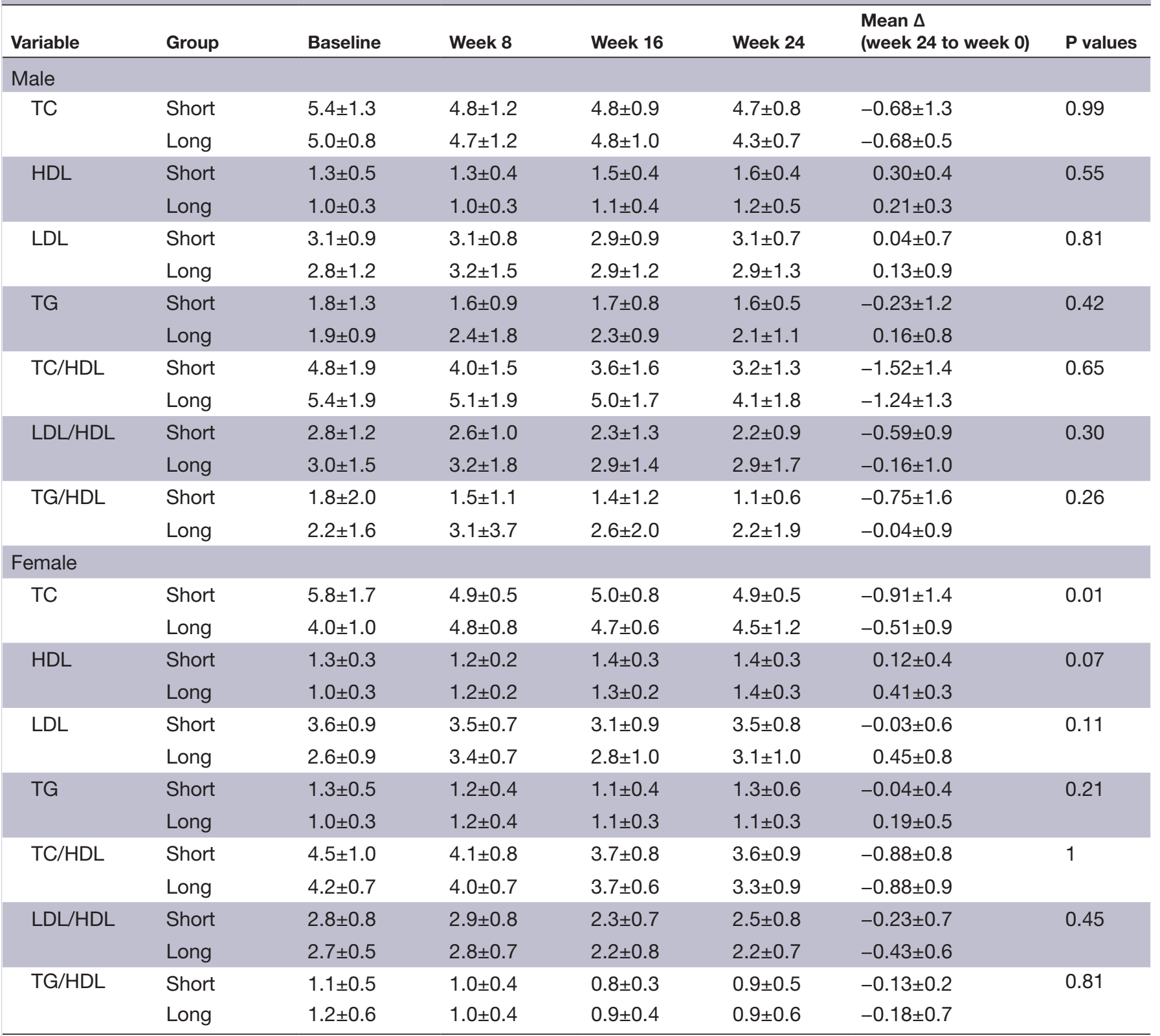

Values are means $\pm S D$ in millimoles per litre of blood. $P$ values represent if the difference in mean change between bouts is statistically significant.

$\Delta$, change; HDL, high-density lipoproteins; LDL, low-density lipoproteins; TC, total cholesterol; TG, triglycerides.

difference could be shown between the exercise regimes for either sex. No study that we are aware of has described this exercise effect in individuals of comparable age and setting and followed for similar time-length as our study.

Males and females in both exercise regimes had an increase in HDL. This finding is consistent with other studies that show exercise affects HDL. ${ }^{31}{ }^{32}$ Specifically, males and females in short-bout exercise regime whose baseline HDL was $<0.9 \mathrm{mmol} / \mathrm{L} \quad(18.5 \%$ and $7.7 \%$, respectively) decreased to 0 . In the $\mathrm{F}_{\mathrm{L}}$ group, those with $\mathrm{HDL}<0.9 \mathrm{mmol} / \mathrm{L}$, also decreased from $15.4 \%$ to $3.8 \%$, and there was no change in the $\mathrm{M}_{\mathrm{L}}$ group. What our study adds and that could be a beneficial interventional approach is that the short sessions appeared more beneficial in improving HDL levels when compared with the traditional regimes. For LDL and TG, the change was marginal. Males and females on long-bout exercise regime had slight rise in both LDL and TG, but there was slight improvement in the short-bout exercise participants.

\section{Effect on lipid ratios}

For lipid ratios, two-thirds of $\mathrm{M}_{\mathrm{S}}$ and $\mathrm{F}_{\mathrm{S}}$ group showed reduced TC/HDL $\left(<5.0\right.$ and $<4.5$, respectively). In $\mathrm{M}_{\mathrm{L}}$, it was a quarter, and in $\mathrm{F}_{\mathrm{L}}$, it was four-fifths. Previously, it has been suggested that intermittent exercise regimes may actually be more beneficial than current exercise regimes in regulation of attributes such as blood pressure 
Table 3 Linear regressions for cardiometabolic variables (controlling for sex)

\begin{tabular}{|c|c|c|c|c|}
\hline $\begin{array}{l}\text { Mean change, } \\
\text { long }\end{array}$ & Coefficient & SE & $P>|t|$ & $95 \% \mathrm{Cl}$ \\
\hline FBG & -0.36 & 0.25 & 0.15 & -0.87 to 0.14 \\
\hline TC & 0.75 & 0.36 & 0.05 & 0.15 to 1.48 \\
\hline HDL & 0.11 & 0.11 & 0.33 & -0.11 to 0.33 \\
\hline LDL & 0.29 & 0.23 & 0.20 & -0.17 to 0.75 \\
\hline TG & 0.30 & 0.24 & 0.22 & -0.18 to 0.78 \\
\hline TC/HDL & 0.13 & 0.34 & 0.70 & -0.56 to 0.82 \\
\hline LDL/HDL & 0.10 & 0.24 & 0.68 & -0.38 to 0.58 \\
\hline TG/HDL & 0.31 & 0.32 & 0.33 & -0.33 to 0.95 \\
\hline
\end{tabular}

FBG, fasting blood glucose; HDL, high-density lipoproteins; LDL, low-density lipoproteins; TC, total cholesterol; TG, triglycerides.

and maximal oxygen consumption. ${ }^{33}$ What our study adds is that short exercise regimes are also beneficial in improving TC/HDL among males. Our results, however, show mixed outcomes since for the females, long-bout exercise regime was marginally superior in improving the TC/HDL ratio. This improvement in females differs from Quinn et $a l^{33}$ who found that shorter exercise regimes provide better outcomes, although their study excluded a comparison of TC/HDL. Furtherore, our study period was twice as long (24 weeks vs 12 weeks), and each of our exercise sessions took half the number of minutes (7.5 min vs $15 \mathrm{~min}$ ) adopted by Quinn et $a l^{33}$ which could have contributed to the difference in our findings. Furthermore, since the long-bout exercise regime was less effective compared with the short-bout exercise regime in lowering $\mathrm{TC}$ in females, the difference in results could be due to the long-bout exercise raising HDL marginally higher thereby reducing the TC/ HDL ratio. The decrease in mean TC/HDL in males and females in the two exercise regimes who did not achieve recommended levels was similar. With regard to TC/HDL ratio, the effect of the two exercise regimes could not be differentiated, and the apparent dissimilarity in the effect among females was not supported when controlling for sex. Thus, the effect on TC/HDL by the two exercise regimes was similar in both males and female.

LDL/HDL had lower change in rate throughout the study period. However, the overall trend was similar in both sexes from the two exercise regimes. Half of $\mathrm{M}_{\mathrm{S}}$ group with baseline values of $>3.5$ showed lower ratios. Slightly less than half of $\mathrm{M}_{\mathrm{L}}$ regime participants also showed LDL/HDL $\leq 3.5$. There was no difference between the groups in overall mean change of LDL/HDL for the duration of the study. The exercises regimes also had comparable mean values in those not reaching the recommended LDL/HDL ranges. Fifty per cent of $F_{S}$ group that started with high values of LDL/HDL reached the recommended threshold, compared with about two-thirds in the $\mathrm{F}_{\mathrm{L}}$ group. Thus, shorter exercise regime was more favourable in LDL/HDL regulation in males, although females still attained satisfactory results since drop in LDL/HDL for traditional group was not statistically superior to that in experimental group. Based on TC/HDL and LDL/ HDL, it is apparent the two exercise regimes had similar reduction of cardiovascular disease risk. This could also address the recent finding, which our baseline data also support, that majority of Kenyans aged above 50 years are at cardiovascular disease risk based on combination of various lipid ratios. ${ }^{30}$ Regardless of gender, all would thus benefit similarly, whether from short or long sessions of exercise. Overall, comparison of these ratios suggest that no difference exists in reduction of metabolic syndrome risk in individuals in either of these exercise regimes.

\section{Blood glucose effects}

FBG levels decreased for all groups. In $\mathrm{M}_{\mathrm{S}}$ group, participants with FBG $>5.5 \mathrm{mmol} / \mathrm{L}$ decreased from $40.7 \%$ to $11.1 \%$, while in the $\mathrm{M}_{\mathrm{L}}$ from $33.3 \%$ to 3.7 . Absolute $\mathrm{FBG}$ mean change, however, was similar between the groups, and participants retaining prediabetic-to-diabetic values were less in $\mathrm{M}_{\mathrm{S}}$ and $\mathrm{F}_{\mathrm{S}}$ than in the $\mathrm{M}_{\mathrm{L}}$ and $\mathrm{F}_{\mathrm{L}}$ groups. In the $\mathrm{F}_{\mathrm{S}}$ group, all participants with prediabetic-to-diabetic baseline FBG levels $(46.2 \%)$ achieved normal levels after the 24 weeks. In the $\mathrm{F}_{\mathrm{L}}$, this was from $42.3 \%$ to $11.5 \%$. The mean change in absolute FBG values between baseline and end-point were similar for $\mathrm{F}_{\mathrm{S}}$ and $\mathrm{F}_{\mathrm{L}}$ groups. Regression analysis controlling for gender showed no significant difference between the short-duration and long-duration bouts of exercise in FBG mean change. The view that longer exercise sessions among the males and the shorter regime for the females were superior based on percentage change (alone) therefore failed to hold further. This, coupled with the observation of no difference in mean change for FBG after 24 weeks for both sexes from the two different exercise regimes, supports our hypothesis that these exercise regimes have similar effects. Thus, shorter/intermittent exercise sessions lasting $<10$ min each regulate elevated FBG to the same extent as the longer traditional sessions lasting $>30$ min per session, as long as the cumulative exercise times are similar.

\section{Limitations}

The design of the protocol may have caused some limitations. Our sample was recruited through print advertisement, which self-selection may have favoured those able to read, compromising inference to the general elderly population. Furthermore, the design could not allow for blinding, depended on subjective health assessment at recruitment with only a physical examination the only form of objective verification, and did not consider lifestyle behaviours such as smoking history and diet, which could have affected health status of participants. These potential limitations may have confounded our results and affected generalisation.

\section{CONCLUSION}

An exercise regime of accumulated short bouts lasting $<10$ min shows improvement of cardiometabolic 
measurements among sedentary adults aged $\geq 50$ years comparable with the currently advocated longer sessions that last $>30$ min each, as long as the intensity and cumulative exercise times are similar.

Contributors KM helped in designing protocol, data collection, analysis and writing. Both KT and NBP helped with designing of protocol and writing the manuscript.

Funding This research was supported by the Consortium for Advanced Research Training in Africa (CARTA). CARTA is jointly led by the African Population and Health Research Center (APHRC) and the University of the Witwatersrand and funded by the Wellcome Trust (UK) (grant no: 087547/Z/08/Z), the Department for International Development (DfID) under the Development Partnerships in Higher Education (DelPHE), the Carnegie Corporation of New York (grant no: B 8606), the Ford Foundation (grant no: 1100-0399), Google.Org (grant no: 191994),Sida (grant no: 54100029), MacArthur Foundation (grant no:10-95915-000-INP) and British Council.

\section{Competing interests None declared.}

\section{Patient consent Obtained.}

Ethics approval The study was approved by the joint Moi Teaching and Referral Hospital and Moi University Research Ethics Committee in Eldoret, Kenya. Ethical approval number: MTRH-MU IREC 0001242.

Provenance and peer review Not commissioned; externally peer reviewed.

Open Access This is an Open Access article distributed in accordance with the terms of the Creative Commons Attribution (CC BY 4.0) license, which permits others to distribute, remix, adapt and build upon this work, for commercial use, provided the original work is properly cited. See: http://creativecommons.org/ licenses/by/4.0/

Published by the BMJ Publishing Group Limited. For permission to use (where not already granted under a licence) please go to http://www.bmj.com/company/ products-services/rights-and-licensing/

\section{REFERENCES}

1. World Health Organization. Global recommendations on physical activity for health. Geneva: World Health Organization, 2010.

2. Ward BW, Clarke TC, Nugent CN. Early release of selected estimates based on data from the 2015 National Health Interview Survey.

Maryland, United States: National Center for Health Statistics, 2016.

3. Nambaka JE, Kamau J, Amusa LO, et al. Factors influencing participation in physical exercise by the elderly in Eldoret West District, Kenya. Afr J Phys Health Edu Recreat Dance 2011;17:462-72.

4. Jakicic JM, Wing RR, Butler BA, et al. Prescribing exercise in multiple short bouts versus one continuous bout: effects on adherence, cardiorespiratory fitness, and weight loss in overweight women. Int J Obes Relat Metab Disord 1995;19:893-901.

5. Marcus BH, King TK, Clark MM, et al. Theories and techniques for promoting physical activity behaviours. Sports Med 1996;22:321-31.

6. Trost SG, Owen N, Bauman AE, et al. Correlates of adults' participation in physical activity: review and update. Med Sci Sports Exerc 2002;34:1996-2001.

7. Murias JM, Kowalchuk JM, Paterson DH. Time course and mechanisms of adaptations in cardiorespiratory fitness with endurance training in older and young men. J Appl Physiol 2010;108:621-7.

8. Paterson DH, Warburton DE. Physical activity and functional limitations in older adults: a systematic review related to Canada's physical activity guidelines. Int J Behav Nutr Phys Act 2010;7:38.

9. Paterson DH, Cunningham DA, Koval JJ, et al. Aerobic fitness in a population of independently living men and women aged 55-86 years. Med Sci Sports Exerc 1999;31:1813-20.

10. Paterson $\mathrm{DH}$, Jones GR, Rice CL. Ageing and physical activity: evidence to develop exercise recommendations for older adults. Can J Public Health 2007;98(Suppl 2):S69-108.
11. Physical Activity Guidelines Advisory Committee (PAGAC). Physical activity guidelines advisory committee report. Washington, DC: US Department of Health and Human Services, 2008.

12. DeBusk RF, Stenestrand U, Sheehan M, et al. Training effects of long versus short bouts of exercise in healthy subjects. Am J Cardiol 1990;65:1010-3.

13. Picorelli AM, Pereira LS, Pereira DS, et al. Adherence to exercise programs for older people is influenced by program characteristics and personal factors: a systematic review. J Physiother 2014;60:151-6.

14. Church TS, Earnest CP, Skinner JS, et al. Effects of different doses of physical activity on cardiorespiratory fitness among sedentary, overweight or obese postmenopausal women with elevated blood pressure: a randomized controlled trial. JAMA 2007;297:2081-91.

15. Lee DC, Artero EG, Sui $X$, et al. Mortality trends in the general population: the importance of cardiorespiratory fitness. $J$ Psychopharmacol 2010;24(4 Suppl):27-35.

16. Lee IM, Bauman AE, Blair SN, et al. Annual deaths attributable to physical inactivity: whither the missing 2 million? Lancet 2013;381:992-3.

17. Bauman A, Lewicka M, Schöppe S. The health benefits of physical activity in developing countries. Geneva: World Health Organization, 2005.

18. Yu R, Yau F, Ho S, et al. Cardiorespiratory fitness and its association with body composition and physical activity in Hong Kong Chinese women aged from 55 to 94 years. Maturitas 2011;69:348-53.

19. Blair SN, Kohl HW, Gordon NF, et al. How much physical activity is good for health? Annu Rev Public Health 1992;13:99-126.

20. Pate RR. Physical activity and public health. JAMA 1995;273:402-7.

21. Murphy MH, Blair SN, Murtagh EM. Accumulated versus continuous exercise for health benefit: a review of empirical studies. Sports Med 2009;39:29-43.

22. Linke SE, Gallo LC, Norman GJ. Attrition and adherence rates of sustained vs. intermittent exercise interventions. Ann Behav Med 2011;42:197-209.

23. Macfarlane DJ, Taylor LH, Cuddihy TF. Very short intermittent vs continuous bouts of activity in sedentary adults. Prev Med 2006;43:332-6.

24. Millán J, Pintó X, Muñoz A, et al. Lipoprotein ratios: physiological significance and clinical usefulness in cardiovascular prevention. Vasc Health Risk Manag 2009;5:757-65.

25. Salazar MR, Carbajal HA, Espeche WG, et al. Relation among the plasma triglyceride/high-density lipoprotein cholesterol concentration ratio, insulin resistance, and associated cardiometabolic risk factors in men and women. Am J Cardiol 2012;109:1749-53.

26. Gotto AM, Assmann G, Carmena R. The ILIB lipid handbook for clinical practice: blood lipids and coronary heart disease. 2nd edn. New York, NY: International Lipid Information Bureau, 2000:201.

27. Alikor CA, Emem-Chioma PC. Epidemiology of diabetes and impaired fasting glucose in a rural community of nigerian niger delta region. Niger J Med 2015;24:114-24.

28. Werfalli M, Engel ME, Musekiwa A, et al. The prevalence of type 2 diabetes among older people in Africa: a systematic review. Lancet Diabetes Endocrinol 2016;4:72-84.

29. Sabir AA, Isezuo SA, Ohwovoriole AE. Dysglycaemia and its risk factors in an urban Fulani population of northern Nigeria. West Afr $J$ Med 2011;30:325-30.

30. Christensen DL, Faurholt-Jepsen D, Birkegaard L, et al. Cardiovascular risk factors in rural Kenyans are associated with differential age gradients, but not modified by sex or ethnicity. Ann Hum Biol 2016;43:42-9.

31. Halverstadt A, Phares DA, Wilund KR, et al. Endurance exercise training raises high-density lipoprotein cholesterol and lowers small low-density lipoprotein and very low-density lipoprotein independent of body fat phenotypes in older men and women. Metabolism 2007;56:444-50.

32. Sarzynski MA, Burton J, Rankinen T, et al. The effects of exercise on the lipoprotein subclass profile: A meta-analysis of 10 interventions. Atherosclerosis 2015;243:364-72.

33. Quinn TJ, Klooster JR, Kenefick RW. Two short, daily activity bouts vs. one long bout: are health and fitness improvements similar over twelve and twenty-four weeks? J Strength Cond Res 2006;20:130-5. 